\title{
Usage of the contactless vector network analysis with varying transmission line geometries
}

\author{
T. Zelder, B. Geck, I. Rolfes, and H. Eul
}

Institut für Hochfrequenztechnik und Funksysteme, Leibniz Universität Hannover, Appelstr. 9A, 30167 Hannover, Germany

\begin{abstract}
The scattering parameters of embedded devices can be measured by means of contactless vector network analysis. To achieve accurate measurement results, the contactless measurement setup has to be calibrated. However, if the substrate material or the planar transmission lines on the substrate changes, a new calibration is necessary. In this paper a method will be examined, which reduces the number of calibration cycles by using a database. Analytical results show that by using this database method, errors occur which depend on the coupling coefficients and on the load impedances of the contactless probes. However, the measurement results show deviations smaller than $7 \%$ in comparison to the conventional vector network analysis, which is sufficient for the most pratical applications.
\end{abstract}

\section{Introduction}

The scattering parameters of embedded devices can be determined by contactless measurement methods described by Sayil et al. (2005), Zelder and Eul (2006), Dudley et al. (1999). A promising method is the contactless vector network analysis proposed by Stenarson et al. (2001), Zelder et al. (2007a). A simplified two-port setup of this contactless measurement technique is illustrated in Fig. 1. The twoport setup consists of a conventional vector network analyzer (VNA) with a source, a switch and four vectorial receivers $m_{1}$ to $m_{4}$. In Fig. 1 the output ports of the VNA are connected to a planar circuit board. In contrast to the conventional setup, contactless probes are connected directly to the receivers. Furthermore, the probes are positioned above the planar transmission lines of the device under test (DUT). In this paper a setup using capacitive probes is described.

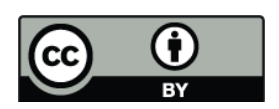

Correspondence to: T. Zelder (zelder@hft.uni-hannover.de)
Zelder and Eul (2006) also used inductive probes, whereas Zelder et al. (2007a) and Yhland and Stenarson (2005) applied loop couplers as contactless probes. After a conventional calibration such as Thru-Reflect-Line (TRL) given by Engen and Hoer (1979), a DUT can be characterized. By moving the probes from one planar DUT to another, the scattering parameters of all DUTs which have equal planar connection lines, can be determined. If the geometry of the planar connection lines or the substrate changes, a new calibration has to be carried out because the coupling coefficients between the contactless probes and the planar transmission lines change. This leads to variations of the calibration error terms. Thus, for each substrate material and each planar transmission line geometry to be measured, a separate calibration substrate must be available. With the introduction of the database method the calibration complexity can be reduced, because only one reference calibration substrate and a database are necessary.

\section{The database method}

In the database, differences of the error terms are stored using on the one hand a planar transmission line of a reference substrate and on the other hand any other planar transmission line. Without loss of generality in this paper, the database method is described using the conventional Short-Open-Load (SOL) calibration algorithm. However, the method can also be applied to other calibration methods like TRL.

At first the well-known one-port error box model of a reflectometer used by Rehnmark (1974) is shown as a flow graph in Fig. 2 . In the graph $a_{1}, b_{1}, a_{\text {DUT }}$ and $b_{\text {DUT }}$ represent the measured waves and the real traveling waves to and from the DUT, respectively. The other parameters illustrate

Published by Copernicus Publications on behalf of the URSI Landesausschuss in der Bundesrepublik Deutschland e.V. 


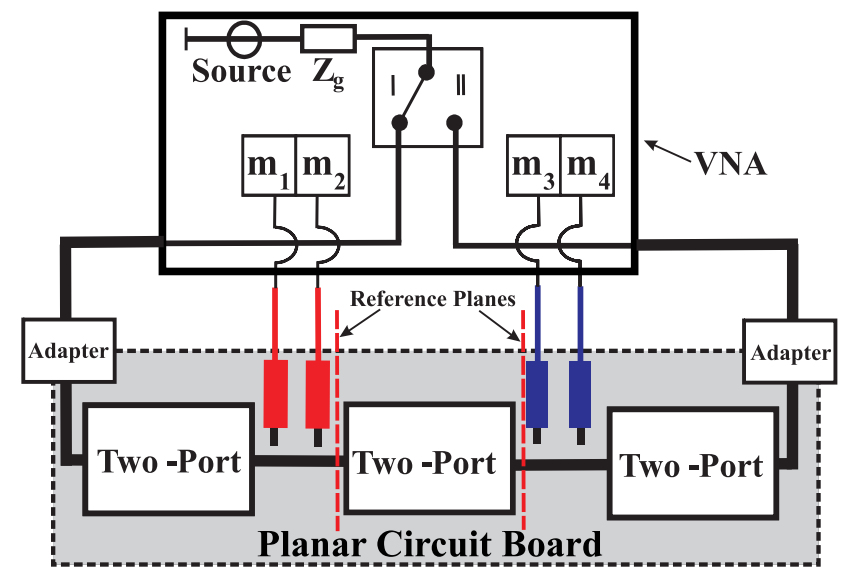

Fig. 1. Contactless vector network analysis setup shown in Zelder et al. (2007b).

the error terms, which can be expressed by a matrix

$[\mathbf{R}]=\left(\begin{array}{cc}R_{11} & R_{12} R_{21} \\ 1 & R_{22}\end{array}\right)$

The error terms $R_{11}, R_{12} R_{21}$ and $R_{22}$ can be determined using three known calibration standards like short, open and load. The ratio $\mu_{x}$ of the measured traveling waves

$\mu_{x}=\frac{b_{1}}{a_{1}}$

for a reflection coefficient $\Gamma_{x}$ in the reference plane can be expressed in dependence of the error terms shown in Eq. (3).

$\mu_{x}=R_{11}+\frac{R_{12} \cdot R_{21} \cdot \Gamma_{x}}{1-R_{22} \cdot \Gamma_{x}}$

Applying Eq. (3) for three calibration standards, the error terms can be calculated, whereby an unknown reflection coefficient $\Gamma_{\text {DUT }}$ can be determined

$\Gamma_{\text {DUT }}=\frac{\mu_{\text {DUT }}-R_{11}}{R_{22} \cdot \mu_{\text {DUT }}+R_{12} \cdot R_{21}-R_{22} \cdot R_{22}}$.

For the database method, the error term matrices $\left[\mathbf{R}_{\mathrm{sub}_{r}}\right],\left[\mathbf{R}_{\mathrm{sub}_{1}}\right],\left[\mathbf{R}_{\mathrm{sub}_{2}}\right], \ldots,\left[\mathbf{R}_{\mathrm{sub}_{n}}\right], \ldots\left[\mathbf{R}_{\mathrm{sub}_{N}}\right]$ according to Eq. (1) using $(\mathrm{N}+1)$ different calibration substrates with different transmission line geometries or substrate permittivities are determined by means of the SOL algorithm and a contactless one-port setup. Thereby, a constant and repeatable probe positioning relative to the DUT and to the transmission line must be warranted. One of the applied substrates can be defined arbitrarily as the reference substrate (index $\operatorname{sub}_{r}$ ). For the database method the following basic approach is arranged

$\left[\mathbf{T}_{\mathrm{sub}_{n}}\right]=\left[\mathbf{T}_{\mathrm{sub}_{r}}\right] \cdot\left[\Delta \mathbf{T}_{\mathrm{sub}_{\mathbf{r n}}}\right]$.

In Eq. (5) the cascade matrix $\left[\mathbf{T}_{\mathrm{sub}_{r}}\right]$ corresponds to the reference error term matrix $\left[\mathbf{R}_{\mathrm{sub}_{r}}\right]$ by

$\left[\mathbf{T}_{\mathrm{sub}_{r}}\right]=\left(\begin{array}{cc}R_{12} R_{21}-R_{11} R_{22} & R_{11} \\ -R_{22} & 1\end{array}\right)$

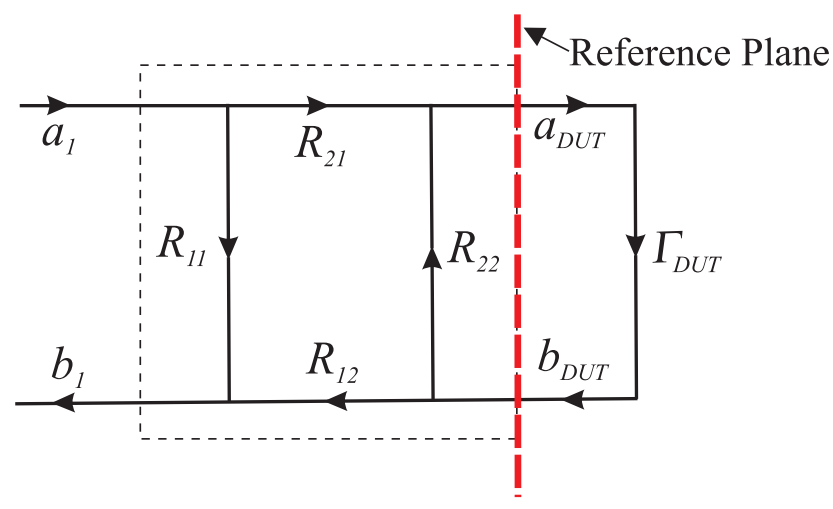

Fig. 2. One-port error box model.

Further more, it is assumed that the cascade matrix $\left[\mathbf{T}_{\mathrm{sub}_{r}}\right]$ multiplied by a matrix $\left[\Delta \mathbf{T}_{\mathrm{sub}_{r n}}\right]$ equals the error term cascade matrix $\left[\mathbf{T}_{\mathrm{sub}_{n}}\right]$ of the substrate $\mathrm{sub}_{n}$. In this case the matrix $\left[\Delta \mathbf{T}_{\mathrm{sub}_{r n}}\right]$ represents the deviations of the error terms between the setup using the reference substrate $\operatorname{sub}_{r}$ and the substrate $\operatorname{sub}_{n}$. The deviation matrices $\left[\Delta \mathbf{T}_{\mathrm{sub}_{r n}}\right]$ can be calculated relatively to the reference transmission line by

$\left[\Delta \mathbf{T}_{\mathrm{sub}_{r n}}\right]=\left[\mathbf{T}_{\mathrm{sub}_{r e f}}\right]^{-1} \cdot\left[\Delta \mathbf{T}_{\mathrm{sub}_{r n}}\right]$

where $\left[\Delta \mathbf{T}_{\mathrm{sub}_{r n}}\right]$ corresponds to

$\left[\Delta \mathbf{T}_{\text {sub }_{r n}}\right]=\left(\begin{array}{cc}\Delta \mathbf{T}_{11, \mathrm{sub}_{r n}} & \Delta \mathbf{T}_{12, \mathrm{sub}_{r n}} \\ \Delta \mathbf{T}_{21, \mathrm{sub}_{r n}} & \Delta \mathbf{T}_{22, \mathrm{sub}_{r n}}\end{array}\right)$.

These deviation matrices $\left[\Delta \mathbf{T}_{\mathrm{sub}_{r n}}\right]$ are stored in the database for all given substrates and transmission lines, respectively.

An alternative way to determine the deviation matrices $\left[\Delta \mathbf{T}_{\mathrm{sub}_{r n}}\right]$ is the usage of a two-tiered calibration. Thereby, the contactless measurement setup is calibrated, for example with a SOL algorithm using the reference substrate. After that, the reference transmission line is replaced by another transmission line, which has a different geometry and/or another substrate material. Then, a second calibration is performed with the changed transmission line and the resulting error term matrix of the second tier is equal to the deviation matrix $\left[\Delta \mathbf{T}_{\mathrm{sub}_{r n}}\right]$.

The database method can be used for example in the quality control of printed circuit boards (PCB), where different transmission line geometries often exist on one board or devices on different substrate materials have to be characterized. Usually, for each change of the transmission line geometry a new calibration has to be carried out. However, by using the database method the contactless measurement setup has to be calibrated only once using the reference substrate. If during the measurement the planar transmission line geometry changes, the error terms can be corrected easily by a software using the database and thus Eq. (5).

Usually the VNA, which is used for generating the database, is different to the VNA which is used during the 


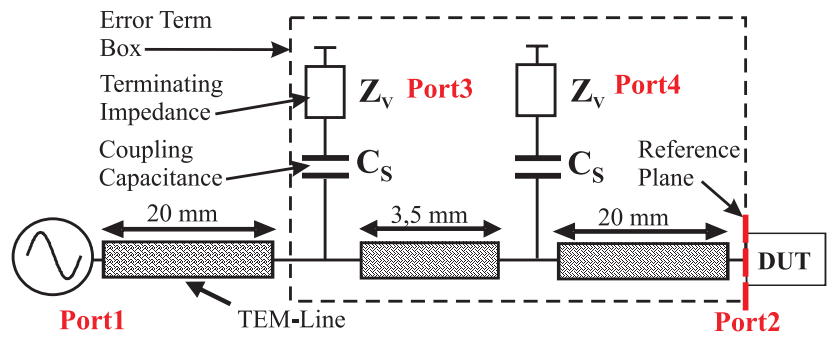

Fig. 3. Analytical one-port model of a contactless measurement setup.

measurement; also the connecting cables between the receivers of the VNA and the contactless probes are different. These facts result in a changed load impedance of the contactless probes. If the database methods are used with a changed load impedance an error occurs. An error estimation using an analytical model will be given in the next section.

\section{Simulation}

\subsection{Analytical model}

In this section the influence of different load impedances $Z_{V}$ of the contactless probes and different coupling coefficients are examined. Therefore, an analytical model is used as illustrated in Fig. 3. In the model a source with a power level of $0 \mathrm{dBm}$ is connected to a coaxial line having conductive loss of $1 \mathrm{~dB} / \mathrm{m}$. Two capacitively coupled probes are used in the one-port scenario which are modeled by two capacitances $C_{S}$. In the simulation results the capacitances for the reference substrate are named $C_{S r}$ and for the measurement substrate $C_{S 1}$. Each capacitance is terminated with the impedance $Z_{V}$, which represents the receiver impedance of the VNA and the interferences of the transmission lines between receiver and probe. The load impedances which are used while the database is set up are named $Z_{V r}$ and the impedances which exist during the measurement of a DUT are named $Z_{V 1}$. The distance between the probes is chosen to be $3.5 \mathrm{~mm}$ and the distance between the last probe and the reference plane is $20 \mathrm{~mm}$. Furthermore, two different substrates are examined. Therefore, two different permittivities $\epsilon_{r, \mathrm{sub} r}=2.0, \epsilon_{r, \text { subl } 1}=2.8$ for the coaxial line between source and DUT are used in the simulation. These values correspond roughly to the effective permittivities of a $50 \Omega$ microstrip line on a Rogers RT5870 and RO4003 substrate. In this paper the coaxial line with the permittivity $\epsilon_{r \text {,subr }}$ is chosen as the reference substrate and the line with the $\epsilon_{r, \text { sub1 }}$ represents the measurement substrate.

\subsection{Analytical results}

Comparing both database methods, exactly the same scattering parameters are obtained, thus, the following results are
Table 1. Capacitances and coupling coefficients at $10 \mathrm{GHz}$.

\begin{tabular}{ccccc}
\hline$C_{S}[\mathrm{fF}]$ & 10 & 15 & 22.5 & 34 \\
\hline$\left|S_{31}\right|[\mathrm{dB}]$ & -30.2 & -26.8 & -23.4 & -20.0 \\
\hline
\end{tabular}

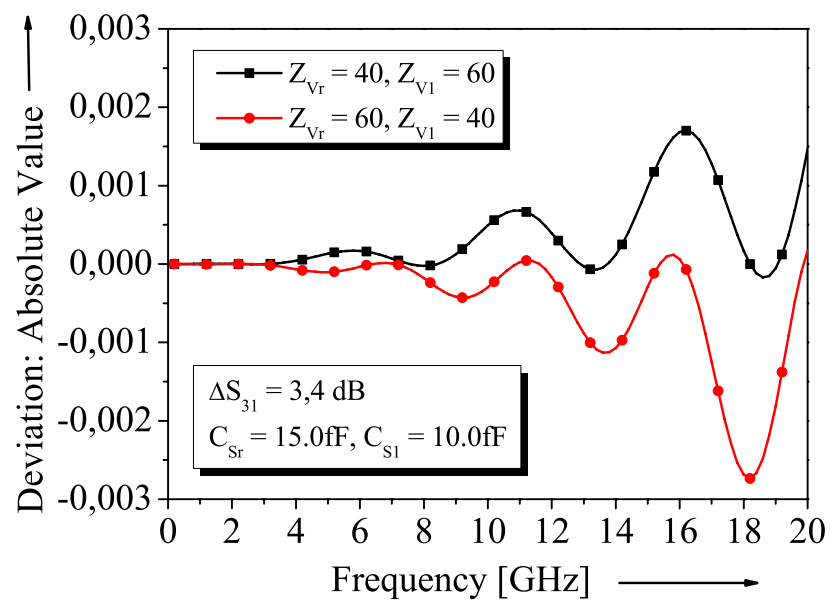

Fig. 4. Deviations of the reflection coefficient in dependency of the the port impedances $Z_{V}$ and the frequency. DUT: $Z_{L}=200 \Omega$.

valid for both methods. In the first analysis the capacitances are chosen to be $C_{S r}=15 \mathrm{fF}$ and $C_{S 1}=10 \mathrm{fF}$ which results in a deviation of $\Delta\left|S_{31}\right|=3.4 \mathrm{~dB}$ and $\Delta \angle\left(S_{31}\right)=61^{\circ}$ at $10 \mathrm{GHz}$. The values of capacitances $C_{S}$ and the coupling factors at $10 \mathrm{GHz}$ are given in Table 1. In Fig. 4, two scenarios are compared, whereas on the one hand the load impedances are chosen to be $Z_{V r}=40 \Omega$ and $Z_{V 1}=60 \Omega$ and on the other hand the impedances are exchanged. The deviations regarding an ideal DUT with an input impedance of $Z_{L}=200 \Omega$ and a constant reflection coefficient of $S_{11}=0.6$ are smaller than $\pm 0.5 \%$ up to $20 \mathrm{GHz}$. The phase deviations which are not illustrated are smaller than $\pm 0.12^{\circ}$.

The increase of the errors in dependence of the difference of the load impedances $\Delta Z=Z_{V 1}-Z_{V r}$ is shown in Fig. 5. For this examination the capacitances are chosen to be $C_{S r}=1 \mathrm{fF}$ and $C_{S 1}=10 \mathrm{fF}$. In Fig. 5 it is illustrated that the error is zero if $\Delta Z=0$. With an increasing $\Delta Z$ the errors also increase. In Fig. 6 the influence of the parameter $S_{31}$ is examined. Using different substrates the complex coupling coefficients depend on the transmission line geometry, its permittivity and the vertical, and horizontal probe positions. Three scenarios are examined, where for all scenarios the same deviation of the coupling coefficient of about $\Delta\left|S_{31}\right|=3.4 \mathrm{~dB}$ exists. Figure 6 makes it obvious that the errors increase if the coupling coefficients $\left|S_{31}\right|$ increase. The reason for that is the increasing interaction between the probes and the main transmission line. Thus, there must be a tradeoff, because low coupling coefficients result 


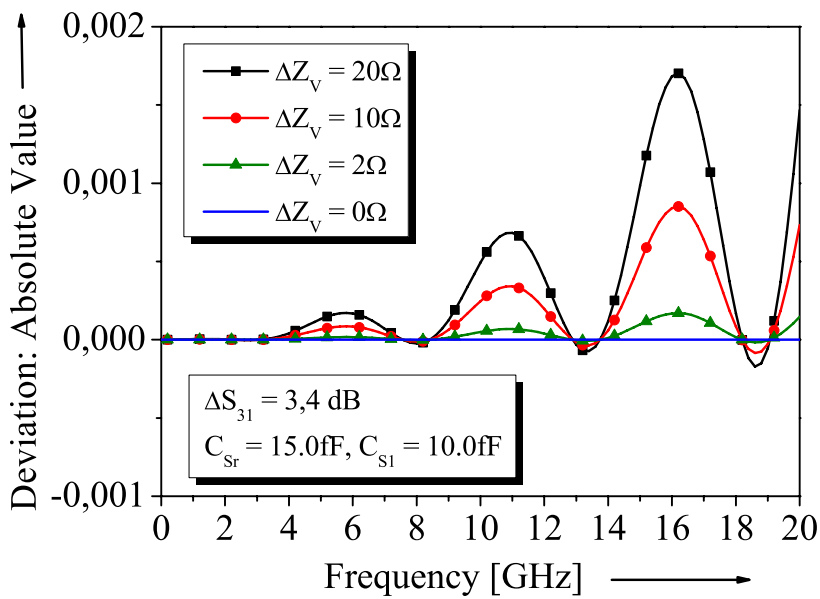

Fig. 5. Deviations of the reflection coefficient in dependency of the differences of the port impedances $\Delta Z$ and the frequency. DUT: $Z_{L}=200 \Omega$.

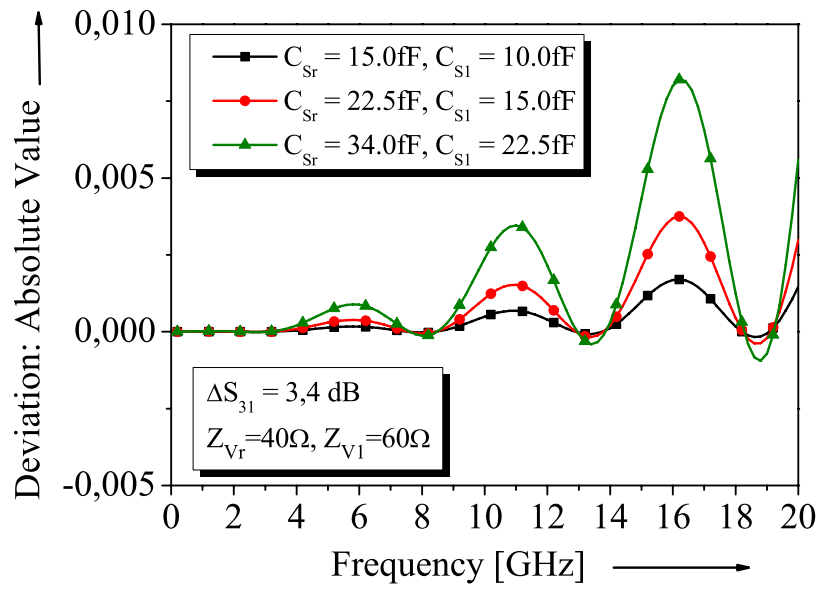

Fig. 6. Deviations of the reflection coefficient in dependency of the coupling capacitances and the frequency. DUT: $Z_{L}=200 \Omega$.

in small errors, however, they also reduce the dynamic range of the contactless measurement. Furthermore, in Figs. 7 and 8 the deviations are separated into absolute error values and phase errors and are shown in dependence of the reflection coefficient. It is obvious that the deviation increases for high reflection coefficients. For the phase deviations the error increases with decreasing reflection coefficients such as the absolute error if it is depicted in percent. In Fig. 9 the deviations depending on the differences $\Delta\left|S_{31}\right|$ of the coupling coefficients using either the reference or the measurement substrate are shown. By adjusting the capacitances $C_{S r}$ and $C_{S 1}$, the differences of the coupling coefficients $\Delta\left|S_{31}\right|$ are changed between $3.4 \mathrm{~dB}$ and $10.2 \mathrm{~dB}$. As shown in Fig. 9, the errors increase with an increasing $\Delta\left|S_{31}\right|$. But even if a strong coupling of about $-20 \mathrm{~dB}\left(C_{S r}=34 \mathrm{fF}\right)$ and a difference of about $\Delta\left|S_{31}\right|=10.2 \mathrm{~dB}$ exist, the measurement error of a $200 \Omega$ load

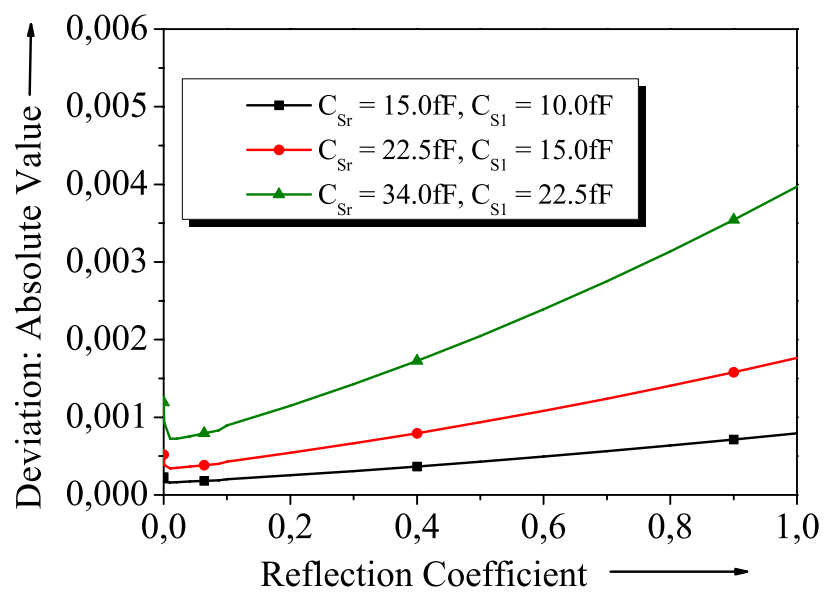

Fig. 7. Deviations of the reflection coefficient in dependency of the coupling capacitances at $10 \mathrm{GHz}$.

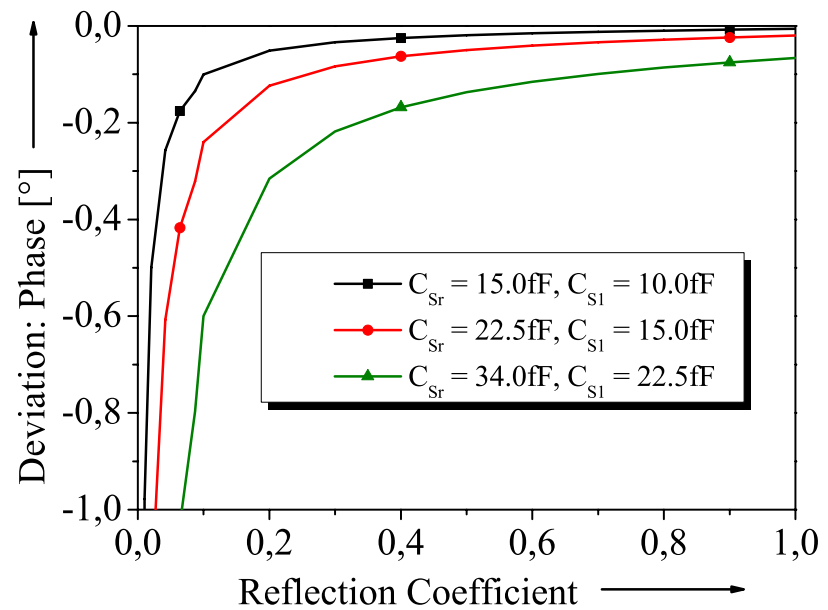

Fig. 8. Deviations of the reflection coefficient in dependency of the coupling coefficient $S_{31}$ at $10 \mathrm{GHz}$.

is smaller than $\pm 2,5 \%$ and $\pm 1^{\circ}$. Figure 10 shows that a high coupling coefficient for the reference substrate as well as for the measurement substrate is even worse. For the chosen coupling coefficients it is insignificant that the probes above the reference substrate or above the measurement substrate have a higher coupling. It can be summarized that using the database method a small error occurs. The errors decrease with decreasing differences of the load impedances $\Delta Z$ of the contactless probes, with decreasing the coupling coefficients $S_{31}$ and $S_{41}$ as well as with decreasing differences of the coupling coefficients $\Delta S_{31}$ and $\Delta S_{41}$ of the reference substrate in comparison to the measurement substrate. 


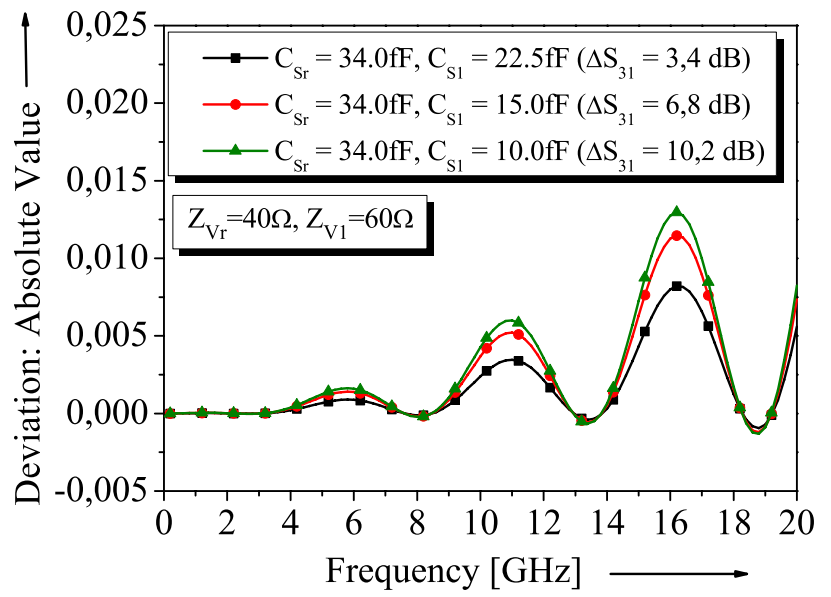

Fig. 9. Deviations of the reflection coefficient in dependency of the differences $\Delta\left|S_{31}\right|$ of the coupling coefficient. DUT: $Z_{L}=200 \Omega$.

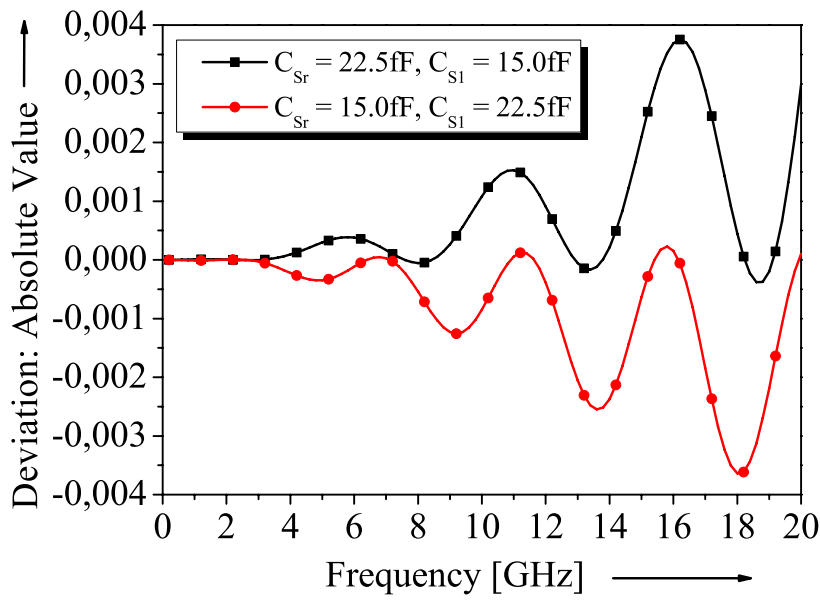

Fig. 10. Deviations of the reflection coefficient in dependency of the order of the capacitances $C_{S r}$ and $C_{S 1}$ and the frequency. DUT: $Z_{L}=200 \Omega$.

\section{Measurement}

\subsection{Measurement setup}

The database method is tested using the measurement setup shown in Fig. 11. For the measurement two small semi-rigid coaxial lines are used as capacitive probes. The inner conductors of the coaxial lines have a diameter of $200 \mu \mathrm{m}$. The probe ends are positioned in a distance of $3.5 \mathrm{~mm}$ from each other and about $50 \mu \mathrm{m}$ above the microstrip lines. Two microstrip lines on Rogers RO4003 $\left(\epsilon_{r}=3.55\right)$ and on RT5870 $\left(\epsilon_{r}=2.33\right)$ are used. The width of the microstrip lines are $1.1 \mathrm{~mm}$ (RO4003) and $1.49 \mathrm{~mm}$ (RT5870) which results in characteristic impedances of about $50 \Omega$. Setting up the reference database, the calibration substrate RT5870 and a VNA (Agilent PNA E8361A) are used. The database method is

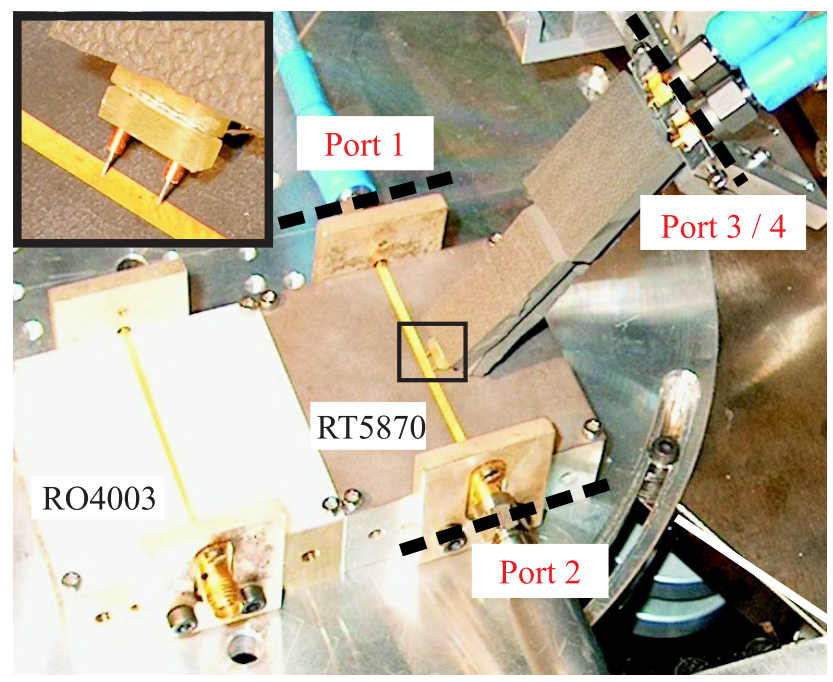

Fig. 11. Contactless measurement setup with capacitive probes.

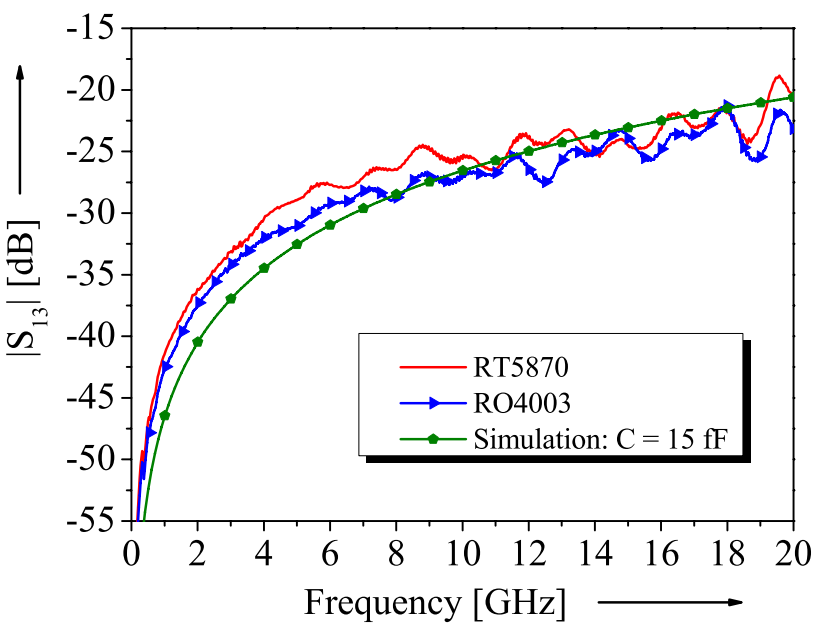

Fig. 12. Comparison of the measured coupling coefficients with a model using a single capacitance.

utilized on the measurement substrate RO4003 using a VNA (Rhode \& Schwarz ZVA24).

\subsection{Measurement results}

At first the characteristics of both the contactless probes and the substrates have been examined. Therefore, a four-port measurement setup is used which is calibrated with a fourport TOSM procedure regarding the coaxial reference planes at port 1 to port 4 . The absolute values of the coupling coefficients using both substrates are plotted over the frequency in Fig. 12. For the RO4003 substrate, the coupling coefficient is about $\left|S_{31}\right|=-26.8 \mathrm{~dB}$ which is comparable with a coupling capacitance of $15 \mathrm{fF}$ as illustrated in Fig. 12. The differences of the coupling coefficients $S_{13}$ and $S_{14}$ using the 


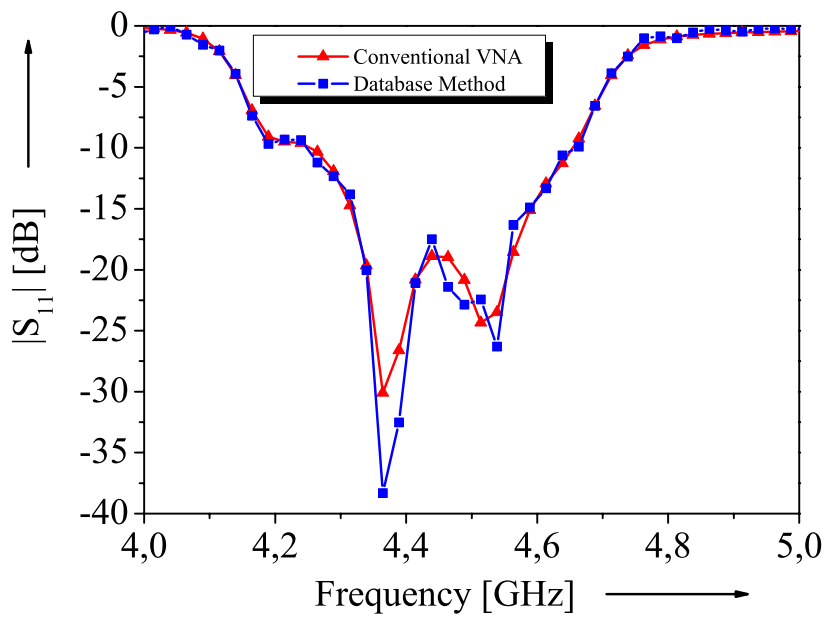

Fig. 13. Measured reflection coefficient of a filter using on the one hand a conventional VNA and on the other hand the contactless database method.

RT5870 and the RO4003 substrate is smaller than $\pm 3.5 \mathrm{~dB}$ in magnitude and $\pm 25^{\circ}$ in phase up to $20 \mathrm{GHz}$. Furthermore, the load impedances of the probes are compared using on the one hand the PNA and on the other hand the ZVA. Over the whole frequency range, the load impedances including the coaxial connecting lines and the receiver impedances vary by $\pm 13 \Omega$ between $1 \mathrm{GHz}$ and $20 \mathrm{GHz}$. The match of the load impedances with respect to a reference impedance of $50 \Omega$ is smaller than $-10 \mathrm{~dB}$. The reflection coefficient of a passband filter is shown in Fig. 13. The measurement results using a conventional VNA and the contactless setup in combination with the database method are in close concordance. The deviations between both measurement results are shown in Figs. 14 and 15. The deviations of the absolute values of the reflection coefficient for high reflective DUTs are smaller than $\pm 7 \%$ and for the phase smaller than $\pm 6^{\circ}$ between $2 \mathrm{GHz}$ and $16 \mathrm{GHz}$. The deviations can be reduced if the measurement results are averaged which has not been done for the results shown in Figs. 13 to 15. With the measurement the deviations are higher than with the simulations, as in the simulations noise, positioning errors, and surrounding influences are neglected.

\section{Conclusions}

Using the contactless vector network analysis it is necessary to recalibrate the setup for any changes of the planar transmission lines which are connected to a DUT. With the database method only one calibration on a reference substrate is necessary to use different transmission line geometries during the measurement. Simulation and measurement results show that the errors of the database method can be neglected in comparison to the errors caused by noise, the

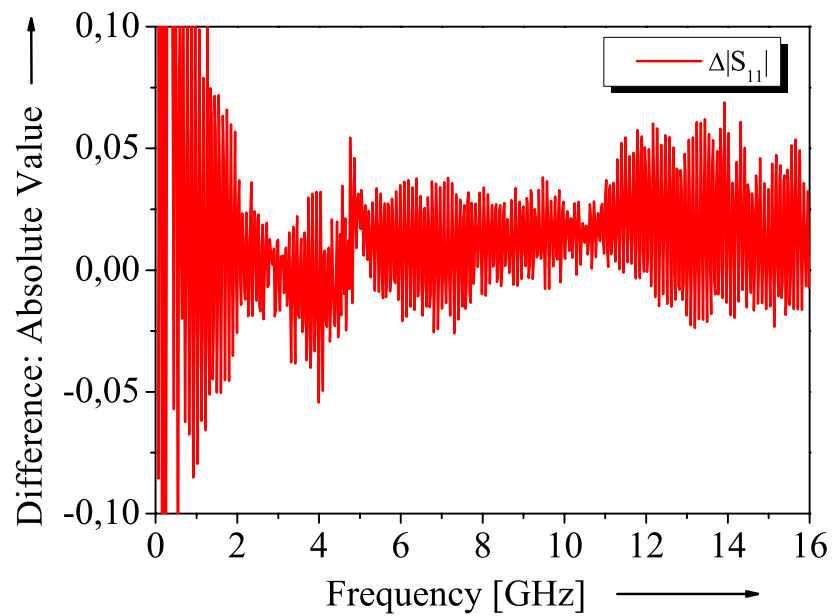

Fig. 14. Deviations of the absolute values of the reflection coefficients of the filter using on the one hand a conventional VNA and on the other hand the contactless database method.

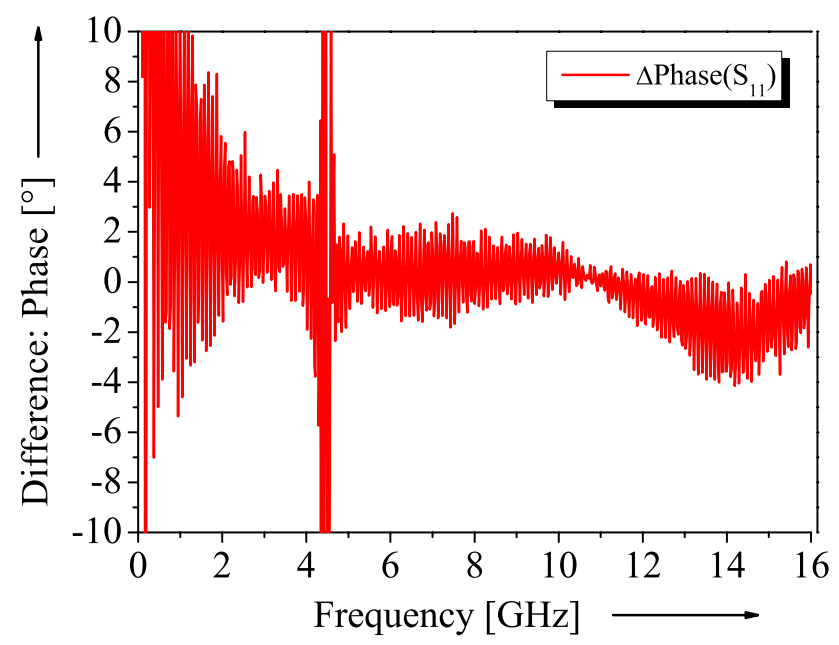

Fig. 15. Deviations of the phase of the reflection coefficients of the filter using on the one hand a conventional VNA and on the other hand the contactless database method.

surroundings, and insufficient reproducibility of the probe positioning.

Acknowledgements. The authors are indebted to Rosenberger Hochfrequenztechnik GmbH \& Co. KG for financial support and to Rhode \& Schwarz GmbH \& Co. KG for sponsoring of the vector network analyzer. 


\section{References}

Dudley, R., Roddie, A., Bannister, D., Gifford, A., Krems, T., and Facon, P.: Electro-optic S-parameter and electric-field profiling measurement of microwave integrated circuits, IEE Proceedings - Science, Measurement and Technology, 146, 117-122, 1999.

Engen, G. and Hoer, C.: Thru-Reflect-Line: An Improved Technique for Calibrating the Dual Six-Port Automatic Network Analyzer, IEEE Transactions on Microwave Theory and Techniques, 27, 987-993, 1979.

Rehnmark, S.: On the calibration process of automatic network analyzer systems, IEEE Transactions on Microwave Theory and Techniques, 22, 457-458, 1974.

Sayil, S., Kerns, D. V. J., and Kerns, S.: Comparison of contactless measurement and testing techniques to a all-silicon optical test and characterization method, IEEE Transactions on Instrumentation and Measurement, 54, 2082-2089, 2005.
Stenarson, J., Yhland, K., and Wingqvist, C.: An in-circuit noncontacting measurement method for S-parameters and power in planar circuits, IEEE Transactions on Microwave Theory and Techniques, 49, 2567-2572, 2001.

Yhland, K. and Stenarson, J.: Noncontacting measurement of power in microstrip circuits, 65th ARFTG Conference Digest, 201-205, 2005.

Zelder, T. and Eul, H.: Contactless network analysis with improved dynamic range using diversity calibration, Proceedings of the 36th European Microwave Conference, 478-481, 2006.

Zelder, T., Geck, B., Wollitzer, M., Rolfes, I., and Eul, H.: Contactless Network Analysis System for the Calibrated Measurement of the Scattering Parameters of Planar Two-Port Devices, Proceedings of the 37th European Microwave Conference, 246-249, 2007a.

Zelder, T., Rabe, H., and Eul, H.: Contactless electromagnetic measuring system using conventional calibration algorithms to determine scattering parameters, Adv. Radio Sci., 5, 427-434, 2007b, http://www.adv-radio-sci.net/5/427/2007/. 